\title{
Artifactual mirrored subclavian artery on ultrasound imaging for supraclavicular block
}

\author{
Peter D. Hebbard, FANZCA
}

Received: 11 March 2009/Accepted: 8 April 2009/Published online: 20 May 2009

(C) Canadian Anesthesiologists' Society 2009

\section{To the Editor,}

I read with interest the letter written by Drs. Saranteas and Karabinis, ${ }^{1}$ who highlighted the important artifactual subclavian artery seen on supraclavicular ultrasound examination using color or power Doppler. I believe, however, that there is an alternative interpretation for the formation of this artifact. The image shown is an example of mirror artifact rather than a reverberation artifact, with the reflecting "mirror" surface being the pleura.

Reverberation artifacts usually originate from prominent interfaces that are roughly parallel to the transducer face and strongly reflect ultrasound back to the transducer. ${ }^{2}$ The ultrasound beam is then reflected at the skin or probe surface and returned into the patient to be reflected for a second (or third) time. The artifact can be observed at multiples of the distance from the strongly reflecting interface and the probe, particularly when superimposed on a dark area of the image (Fig. 1). Typical sources of reverberation include the subcutaneous fat-muscle interface, bowel gas, and the pleura. Multiple reverberation sources may occur and produce very "noisy" ultrasound images. ${ }^{2}$ In the case of a complex structure such as an artery, only the superficial and deep walls parallel to the transducer face would be capable of making reverberation artifacts, and the walls of the first reverberated image would appear twice the distance apart than in the "real "image (each artifactual wall would be independently double the actual distance from the transducer). Blood is generally a poor reflector of ultrasound (blood vessels appear dark and anechoic on ultrasound imaging) making it an unlikely source of reverberation. Color Doppler uses

P. D. Hebbard, FANZCA $(\bowtie)$

Northeast Health Wangaratta, Wangaratta, Australia

e-mail: p.hebbard@bigpond.com

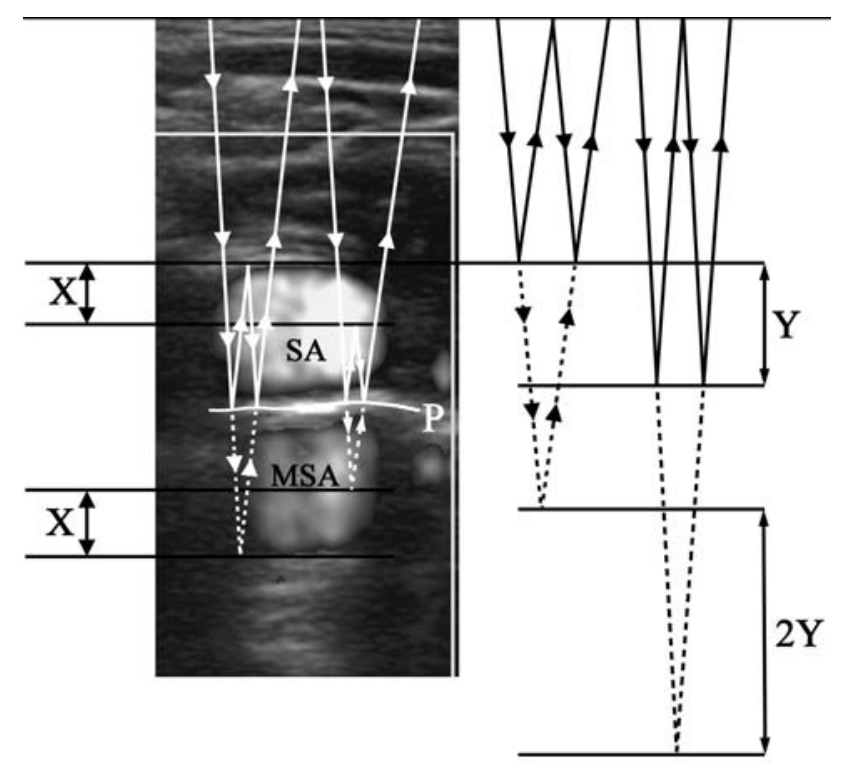

Fig. 1 Power Doppler image of subclavian artery, mirror artifact and superimposed diagram of an ultrasound beam path producing mirror artifact. The real beam path is solid lines and the artifactual path is dashed lines. Note that the distance ' $X$ ' between the mirrored parts of the artery is the same. The diagram on the right shows the beam path for the reverberation artifact. Note how the original distance ' $\mathrm{Y}$ ' between the reverberating surfaces is doubled in the artifact. The line of the pleura $(\mathrm{P})$ is indicated as well as the subclavian artery (SA) and the mirrored subclavian artery (MSA)

higher power ultrasound than normal 2D imaging, however, the returning signal is still relatively weak.

A mirror artifact is caused by reflection of the ultrasound beam by a strong ultrasound reflecting surface that is not necessarily parallel to the transducer face but acts as a mirror. Mirror artifacts may be complex depending on the shape of the "mirror"; however, the mirroring interface is 
always apparent between the two images of the mirrored structure. $^{2}$ In the picture by Drs. Saranteas and Karabinis, ${ }^{1}$ the pleura is seen midway between the images of the subclavian artery and the artifactual artery showing it as the source of the mirrored artifact.

Conflicts of interest None declared.

\section{References}

1. Saranteas T, Karabinis A. Reverberation: source of potential artifacts occurring during ultrasound-guided regional anesthesia. Can J Anesth 2009; 56: 174-5.

2. Gent R. Applied physics and technology of diagnostic ultrasound. Adelaide, Australia: Milner Publishing; 1997. p. 177-220.

\section{Reply}

We read with interest the letter by Dr. Hebbard, who clarified the mechanism whereby ultrasound energy induces double imaging. We believe that understanding the physical properties of ultrasound is the basis for recognizing artifacts and preventing misinterpretation. Although 'mirror' image refers to duplication of a structure, the physical explanation of this phenomenon is based on the reverberation of sounds between strong reflectors that can result in the creation of mirror artifacts.

Ultrasound images are formed by the transducer producing the beam and then detecting the beam as it is reflected from tissue interfaces. The actual depth the image is placed on the screen is based on the period of time from when the transducer generated the pulse. The presupposition is that the ultrasound pulse always travels in a straight line. ${ }^{1,2}$

When sound waves are reflected by a highly reflective and extended surface, the sound signal is scattered, and not all sound waves return directly to the transducer. Rather, they may be reflected by adjacent portions of the specular reflector. In this case, sounds have an increased "round trip time" with respect to the transducer. The distance of the reflector from the probe is calculated on the base of the "time flight" of the returning sonic signal. The delayed arrival of the reflected signal is now interpreted by the transducer as evidence of a reflecting interface further
Fig. 1 Ultrasound signals reflected by pleura do not return to the transducer; they are reverberated on subclavian artery walls, thus leading to a mirror image. $\mathrm{SA}=$ Subclavian Artery; MSA $=$ Mirror Subclavian Artery; P = Pleura; $\mathrm{AW}=$ Anterior Wall of subclavian artery; $\mathrm{PW}=$ Posterior Wall of subclavian artery

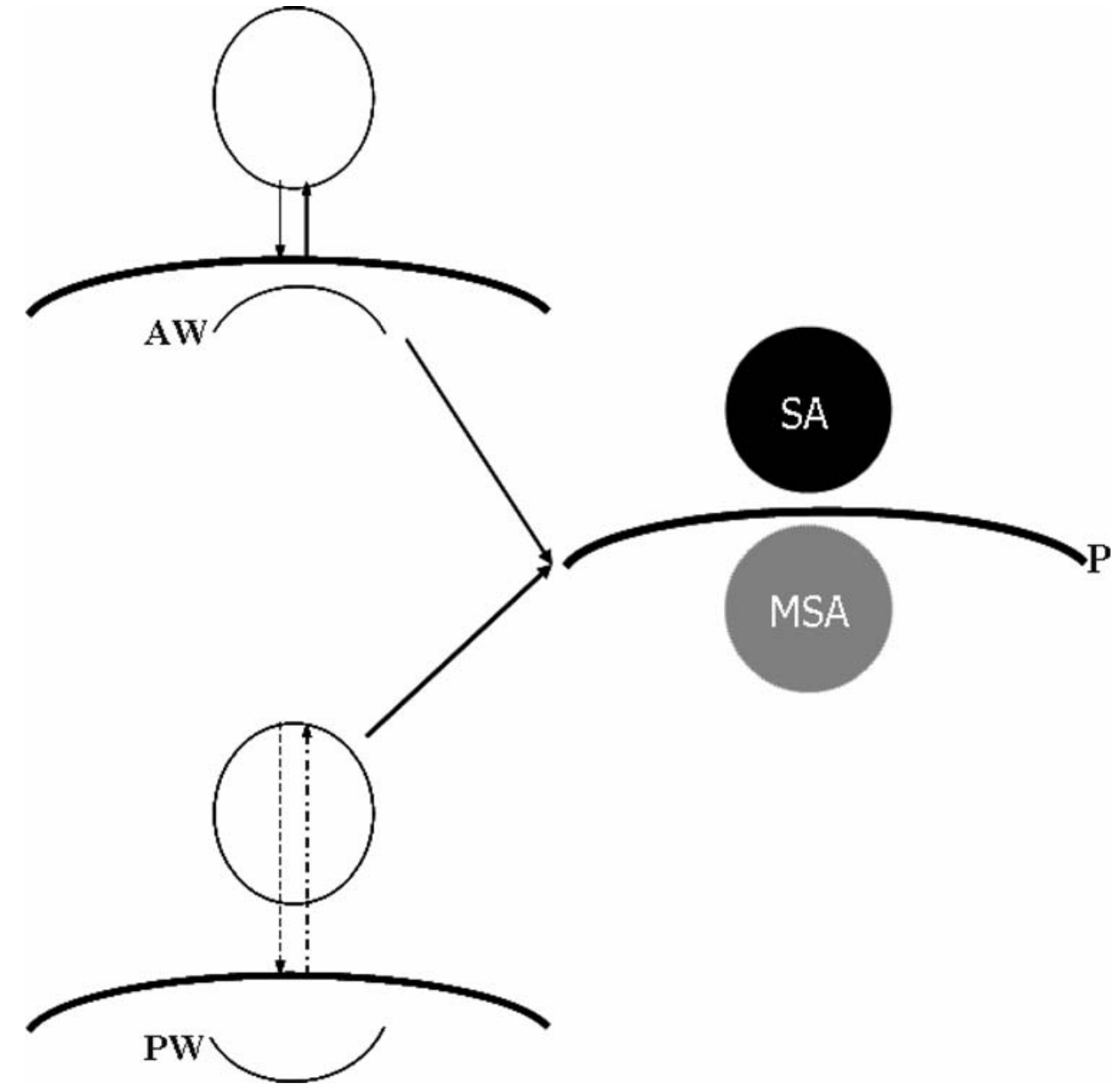


away from the transducer. Structures immediately adjacent to highly reflective acoustic interfaces (pleura) may appear to be duplicated because of reverberation within their walls or as a result of scattering of the sonic signal (Fig. 1). ${ }^{1}$ The recording of information arising from reverberated signals results in the formation of a double image. The false image is commonly inverted as a mirror image, but it also can be misplaced, distorted or entirely projected 'off-axis'. ${ }^{1-3}$

In the case of lung echography, the diaphragm also constitutes a strong reflector. The image gets mapped to the far side of the diaphragm where the lungs are located. In this example, the liver parenchyma, gallbladder, or ascites are mirrored into the thorax with the sharp hyperechoic line of the diaphragm separating them. ${ }^{1-3}$

The mirror image artifact can also occur with the Doppler system. For instance, an image of a vessel and a source of Doppler shifted echoes can be duplicated on the opposite side of a strong reflector such as pleura. The duplicated vessel containing flow could be misinterpreted as an additional vessel. A mirror image of a Doppler spectrum can appear on the opposite side of the baseline when, indeed, flow is unidirectional and should appear only on one side of the baseline. This is an electronic duplication of spectral information due to reverberated ultrasound energy. ${ }^{3-5}$

Using high frequency transducers, reducing energy output, adjusting gain settings, or applying tissue harmonics may abolish this artifact.

Conflicts of interest None declared.

\section{References}

1. Laing FC. Commonly encountered artifacts in clinical ultrasound. Semin Ultrasound 1983; 4: 27-43.

2. Hulsmans FJ, Castelijns JA, Reeders JW, Tytgat GN. Review of artifacts associated with transrectal ultrasound: understanding, recognition, and prevention of misinterpretation. J Clin Ultrasound 1995; 23: 483-94.

3. Kremkau FW, Taylor KJ. Artifacts in ultrasound imaging. J Ultrasound Med 1986; 5: 227-37.

4. Pozniak MA, Zagzebski JA, Scanlan KA. Spectral and color Doppler artifacts. Radiographics 1992; 12: 35-44.

5. Feigenbaum $H$. Physics and instrumentation. In: Feingenbaum $\mathrm{H}$, Armstrong WF, Ryan T, editors. Feigenbaum's echocardiography. Philadelphia: Lippincott Williams \& Willkins; 2005. p. 29-32.

Theodosios Saranteas, MD

Georgia Kostopanagiotou, MD, PhD

Attikon Hospital, University of Athens, Athens, Greece

Andreas Karabinis, MD

General State Hospital of Athens,

Athens, Greece

\section{Commentary}

In the above correspondence items in this issue of Journal, anesthesiologists from different parts of the globe, Drs. Saranteas et al. from Greece and Dr. Peter Hebbard from Australia, discuss two of the many potential artifacts encountered during performance of ultrasound-guided regional anesthesia-reverberation artifact and mirror image artifact. At first glance, the authors seem to be contradicting each other, particularly with reference to Saranteas's original correspondence. ${ }^{1}$ The confusion may have arisen from experts using 'loose' definitions for the word reverberation and for the specifically defined term reverberation artifact.

The word reverberation has been defined as "the persistence of a sound after its source has stopped, caused by multiple reflections of the sound within a closed space", or simply "a re-echoed sound". ${ }^{2}$ Clearly, reverberation could lead to various image phenomena depending on the environment in which the sound travels. During ultrasound imaging, reverberation occurs due to the multiple reflections from an object if the acoustical impedances of tissue layers are significantly different. However, the specific term reverberation artifact is used by many experts when referring to multiple equally-spaced echoes generated when the ultrasound beam re-enters the patient after its reflection back to the transducer from a strong reflector. ${ }^{3}$ Thus, the reflection occurs between the transducer and a strong reflector. On the other hand, the phenomenon of the artifact with an existing structure being duplicated on the other side of a strong reflector is given another specific term, mirror image artifact. In this case, the transducerskin interface is not necessary and an internal reflector produces the image. Nevertheless, some experts rightly describe a mirror image artifact as a "form of reverberation". 4

Strictly speaking, the word reverberation is not synonymous with the term reverberation artifact. Despite this, some still sporadically use the term reverberation artifact interchangeably with the word reverberation. Indeed, the terms reverberation, reverberation artifact, and mirror image artifact have occasionally been used ambiguously in the literature. This point is well illustrated in a recent article, where the authors make the statement, "...one of the more common artifacts is the mirror image. This is a reverberation artifact caused by sound bouncing between multiple structures that are strong specular reflectors...". Similarly, several expert regional anesthesiologists, along with their radiology colleagues, published excellent papers discussing such artifacts. ${ }^{6,7}$ In these publications, what would technically be considered a mirror image artifact 
due to reverberating echoes, is also ambiguously referred to as a "reverberation artifact".

The correspondence items in this issue highlight and remind us that our selection of terminology can be a source of confusion for others. While the word reverberation may be used to describe "the physical explanation of this (mirror image) phenomenon", as Saranteas et al.'s reply suggests, one should use this word carefully to avoid misrepresentation of the defined term reverberation artifacts referred to by Dr. Hebbard.

\section{Conflicts of interest None declared.}

\section{References}

1. Saranteas T, Karabinis A. Reverberation: source of potential artifacts occurring during ultrasound-guided regional anesthesia. Can J Anesth 2009; 56: 174-5.
2. Stein S. Random house college dictionary. New York: Random House; 1979.

3. Hulsmans FJ, Castelijns JA, Reeders JW, Tytgat GN. Review of artifacts associated with transrectal ultrasound: understanding, recognition, and prevention of misinterpretation. J Clin Ultrasound 1995; 23: 483-94.

4. Kremkau FW. Chapter 8: artifacts. In: Kremkau FW, editor. Diagnostic ultrasound principles and instruments. 6th ed. Philadephia: W.B. Sauders; 2002. p. 273-311.

5. Gustavson $S$, Olin JW. Images in vascular medicine. Mirror image artifact. Vasc Med 2006; 11: 175-6.

6. Sites BD, Brull R, Chan VW, et al. Artifacts and pitfall errors associated with ultrasound-guided regional anesthesia. Part II: a pictorial approach to understanding and avoidance. Reg Anesth Pain Med 2007; 32: 419-33.

7. Sites BD, Spence BC, Gallagher J, et al. Regional anesthesia meets ultrasound: a specialty in transition. Acta Anaesthesiol Scand 2008; 52: 456-66.

Ban C. H. Tsui, MD

University of Alberta, Edmonton, Canada 